Research Article

\title{
Facile Solvothermal Synthesis of Hollow BiOBr Submicrospheres with Enhanced Visible-Light-Responsive Photocatalytic Performance
}

\author{
Linrui Hou, Yawei Niu, Fan Yang, Fengyue Ge, and Changzhou Yuan \\ School of Material Science and Engineering, University of Jinan, Jinan 250022, China \\ Correspondence should be addressed to Changzhou Yuan; mse_yuancz@ujn.edu.cn
}

Received 23 June 2019; Revised 27 January 2020; Accepted 4 February 2020; Published 9 March 2020

Academic Editor: Luca Tortora

Copyright (c) 2020 Linrui Hou et al. This is an open access article distributed under the Creative Commons Attribution License, which permits unrestricted use, distribution, and reproduction in any medium, provided the original work is properly cited.

\begin{abstract}
In this work, hierarchical hollow BiOBr submicrospheres (HBSMs) were successfully prepared via a facile yet efficient solvothermal strategy. Remarkable effects of solvents upon the crystallinities, morphologies, and microstructures of the $\mathrm{BiOBr}$ products were systematically investigated, which revealed that the glycerol/isopropanol volumetric ratio played a significant role in the formation of hollow architecture. Accordingly, the underlying formation mechanism of the hollow submicrospheres was tentatively put forward here. Furthermore, the photocatalytic activities of the resulting HBSMs were evaluated in detail with photocatalytic degradation of the organic methyl orange under visible light irradiation. Encouragingly, the as-obtained HBSMs with striking recyclability demonstrated excellent visible-light-responsive photocatalytic performance, which benefits from their large surface area, effective visible light absorption, and unique hollow feature, highlighting their promising commercial application in waste water treatment.
\end{abstract}

\section{Introduction}

In recent years, semiconductor photocatalysis has gradually been recognized as a promising approach to effectively solve the ever-increasing energy shortage and environmental pollution $[1,2]$. Among various photocatalytic materials, $\mathrm{BiOBr}$ is of particular interest thanks to its stability, suitable bang gap, visible-light-response performance, and desirable photocatalytic activities [3-5]. Up till now, numerous pioneer researches have proved that the photocatalytic activities of a semiconductor are hugely dependent upon its size, configuration, and shape $[6,7]$. In this context, the specific microstructures of photocatalysts have been tuned finely to improve their photocatalytic activities [8-10]. Furthermore, the $\mathrm{BiOBr}$-based materials with various shapes including nanoplates, nanosheets (NSs), microspheres, and nanoflakes have been well exploited for photocatalytic applications [11-15]. In particular, the photocatalysts of hollow interiors always display the inimitable advantages in comparison with their solid counterparts, benefiting from the enhanced light-harvesting capacities through the light multiple reflection within the interior cavity, effective separation of photogenerated electron-hole pairs, and larger specific surface area (SSA) [16-19].

The template-assisted approaches constitute a constant focus of researches due to their superiorities in constructing hollow architectures towards a broad range of research fields and industrial processes $[17,20,21]$. For instance, Han et al. prepared the $\mathrm{NiCO}_{2} \mathrm{~S}_{4} / \mathrm{Co}_{9} \mathrm{~S}_{8}$ hollow spheres via the solvothermal method coupled with anion exchange process by using carbon spheres as the sacrificial template [22]. Jia and coworkers synthesized the luminescent $\mathrm{Y}_{2} \mathrm{O}_{3}$ hollow spheres with colloidal melamine formaldehyde template [23]. Also, our group previously synthesized mesoporous hollow $\mathrm{NiCo}_{2} \mathrm{O}_{4}$ submicrospheres towards supercapacitors by applying silica spheres as hard templates [24]. Unfortunately, the hard template-engaged methodologies inevitably suffer from the time-/energy-consuming issues, due to the involved multistep processes, and partial structural collapse with the removal of templates, which thereby stimulates the 
interest in the surfactant-engineered or even template-free fabrication of hollow architectures [25-27]. As for the $\mathrm{BiOBr}$, Zhang et al. synthesized NSs-assembled $\mathrm{BiOBr}$ microspheres with non-close-packed structure via a microwave-assisted solvothermal route, and they exhibited good adsorptive capacity and excellent photocatalytic activity for organic dye [28]. Xia and coworkers prepared the porous hollow $\mathrm{BiOBr}$ spheres with a unique ionic liquid of 1 hexadecyl-3-methylimidazolium bromide [29]. Although enormous advances have been made for the smart synthesis of hollow $\mathrm{BiOBr}$ photocatalysts, there still remains huge research space to further boost their photocatalytic efficiency and expand their applications. Thus, it is highly desirable to develop a simple, green, and efficient avenue to prepare hollow $\mathrm{BiOBr}$ with high photocatalytic degradation performance for organic pollutants.

With the overviews above in mind, herein, we first scalably prepared hollow $\mathrm{BiOBr}$ submicrospheres (HBSMs) by using a simple solvothermal method, where the di-ndecyldimethylammonium bromide (DDAB) acts as Br source and the mixed glycerol (GC)/isopropanol (IP) as the solvent. The key role of the DDAB in the formation of the HBSMs and effects of various solvent systems upon the crystal phases, morphologies, and photocatalytic activities of the obtained $\mathrm{BiOBr}$ samples were systematically investigated. The photocatalytic activities and recyclability of the resulting HBSMs were examined by using methylene orange $(\mathrm{MO})$ as the target pollutant under the Xenon arc lamp irradiation. Besides, the underlying formation mechanism of the HBSMs as well as their photocatalytic degradation mechanism for the dye $\mathrm{MO}$ was tentatively shed light upon here.

\section{Experimental}

2.1. Materials. $\mathrm{Bi}\left(\mathrm{NO}_{3}\right)_{3} \cdot 5 \mathrm{H}_{2} \mathrm{O}, \mathrm{DDAB}$, Degussa $\mathrm{P} 25 \mathrm{TiO}_{2}$ (P25), GC, and IP of analytical grade were all purchased from Sinopharm Chemical Reagent Co., Ltd, and used as received without further purification.

2.2. Preparation of the BiOBr Samples. Hollow BiOBr submicrospheres (HBSMs) were prepared via a facile solvothermal method, and corresponding synthetic route was presented in Figure 1. In a typical synthesis of the HBSMs, $0.488 \mathrm{~g}$ of $\mathrm{DDAB}$ was dissolved into a solution containing $20 \mathrm{~mL}$ of GC and $20 \mathrm{~mL}$ of IP in an ultrasonic bath. Afterwards, $0.485 \mathrm{~g}$ of $\mathrm{Bi}\left(\mathrm{NO}_{3}\right)_{3} \cdot 5 \mathrm{H}_{2} \mathrm{O}$ was added to the above solution, and then the mixed solution was kept at $50^{\circ} \mathrm{C}$ for $30 \mathrm{~min}$ under ultrasonication. Subsequently, the mixture was transferred into a Teflon-lined autoclave $(50 \mathrm{~mL})$ and kept at $180^{\circ} \mathrm{C}$ for $16 \mathrm{~h}$. After being cooled to room temperature naturally, the resulting precipitate was collected by centrifugation, washed, and further dried at $80^{\circ} \mathrm{C}$ for $6 \mathrm{~h}$ before further characterizations. For comparison, another two $\mathrm{BiOBr}$ samples were further obtained with similar procedure just by using the GC or IP as the sole solvent, and the corresponding products were denoted as $\mathrm{BiOBr}-\mathrm{GC}$ and $\mathrm{BiOBr}$-IP for convenience. The detailed synthetic parameters were shown in Table 1.

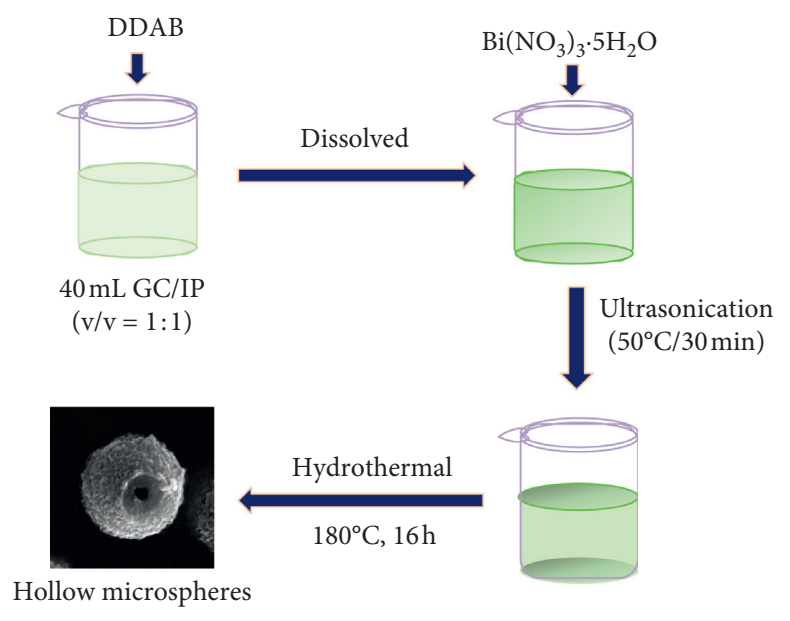

FIgure 1: Synthesis flowchart for HBSMs.

2.3. Materials Characterizations. The crystalline phases of samples were determined on powder X-ray diffractometer (XRD, Rigaku Ultima IV, Japan). Typical morphologies and microstructures of the samples were characterized by using field-emission scanning electron microscopy (FESEM, JEOL-6300F), transmission electron microscopy (TEM), high-resolution TEM (HRTEM), scanning TEM (STEM), and selected area electron diffraction (SAED) (JEOL JEM 2100 system). The Brunauer-Emmett-Teller (BET) SSA of the samples was calculated from $\mathrm{N}_{2}$ adsorption-desorption isotherms measured on a surface area analyzer (TriStar II 3020) at liquid nitrogen temperature. UV-vis diffuse-reflectance spectra were performed to determine the band gap energy $\left(E_{g}\right)$ of the photocatalysts by using a Hitachi U-3010 spectrophotometer. The total organic carbon analysis (TOC) was obtained on Shimadzu TOC-VCPN. X-ray photoelectron spectroscopy (XPS) was recorded by a PHI 5000 X-ray photoelectron spectrometer equipped with an $\mathrm{Al} \mathrm{K \alpha}$ $(\sim 1486.6 \mathrm{eV})$ radiation source, and the spectra were well fitted using the XPSPEAK41 software.

2.4. Photocatalytic Evaluation. Photocatalytic activities of the samples were evaluated by photocatalytic degradation of the $\mathrm{MO}$ under visible light irradiation. A $350 \mathrm{~W}$ Xe arc lamp with a cutoff filter $(\lambda>400 \mathrm{~nm})$ was furnished as the visible light source in the photoreaction system. Typically, $0.3 \mathrm{~g}$ of the photocatalyst was dispersed in $100 \mathrm{~mL}$ of the $\mathrm{MO}$ aqueous solution with an initial concentration of $2 \times 10^{-5} \mathrm{M}$ in a quartz reactor. Prior to illumination, the suspension solution was stirred magnetically in dark for $60 \mathrm{~min}$ to ensure an adsorption/desorption equilibrium. Afterwards, the solution was exposed to visible light irradiation under magnetic stirring. At given time intervals, an aliquot of $3 \mathrm{~mL}$ of suspension solution was drawn and then centrifuged to remove the particles $(4000 \mathrm{rpm}, 10 \mathrm{~min})$. The optical absorption spectra for the supernatant solution were recorded by a doublebeam Shimadzu UV-3600 spectrophotometer (Japan).

2.5. Active Species Trapping Experiment. To determine the active species produced in the photocatalytic process, such as hydroxyl radical $(\cdot \mathrm{OH})$, superoxide radical $\left(\cdot \mathrm{O}_{2}^{-}\right)$, and hole 
TABLE 1: Summary of detailed parameters of control experiments and corresponding structural data of the $\mathrm{HBSMs}, \mathrm{BiOBr}-\mathrm{GC}$, and $\mathrm{BiOBr}-$ IP products.

\begin{tabular}{|c|c|c|c|c|c|}
\hline Samples & Reaction medium & Shape and crystal size (nm) & Band edge (nm) & $E_{g}(\mathrm{eV})$ & $S_{\text {BET }}\left(\mathrm{m}^{2} \cdot \mathrm{g}^{-1}\right)$ \\
\hline HBSMs & $20 \mathrm{~mL} \mathrm{GC}+20 \mathrm{~mL} \mathrm{IP}$ & Spheres (13.49) & 467 & 2.66 & $\sim 55.8$ \\
\hline $\mathrm{BiOBr}-\mathrm{GC}$ & $40 \mathrm{~mL} \mathrm{GC}$ & Spheres (7.23) & 454 & 2.73 & $\sim 17.3$ \\
\hline BiOBr-IP & $40 \mathrm{~mL}$ IP & Sheets (14.52) & 485 & 2.56 & $\sim 0.94$ \\
\hline
\end{tabular}

$\left(\mathrm{h}^{+}\right)$, the isopropyl alcohol (IPA), benzoquinone (BQ), and ethylenediaminetetraacetate (EDTA-2Na) with concentration of $1 \mathrm{mM}$ are separately introduced into the photocatalytic reaction solution $[30,31]$.

\section{Results and Discussion}

3.1. Structural and Physicochemical Characterizations. In this work, a facile solvothermal methodology was devised to prepare the HBSMs with a mixed solvent of GC and IP $(1: 1$, $\mathrm{v}: \mathrm{v})$. As is well known, the solvents generally render certain effects on the crystal growth and even phase composition of a semiconductor over the solvothermal process [32-34]. To this end, the GC or IP was also adopted as a single solvent to synthesize $\mathrm{BiOBr}$ samples, as depicted in Table 1.

The XRD technique was employed to determine their crystalline structures and phase compositions. Figure 2 comparably exhibits the XRD reflections of the as-obtained HBSMs, BiOBr-GC, and BiOBr-IP samples. It can be found that the solvent did not change the crystal structures of samples at all. All the distinctive reflections at 2 theta values of $10.9^{\circ}, 21.9^{\circ}, 25.2^{\circ}, 31.7^{\circ}, 32.2^{\circ}, 33.1^{\circ}, 39.4^{\circ}, 44.7^{\circ}$, $46.2^{\circ}, 50.7^{\circ}, 56.1^{\circ}$, and $57.1^{\circ}$ can be perfectly identified as the (001), (002), (101), (102), (110), (003), (112), (004), (200), (104), (114), and (212) crystal planes of the tetragonal $\mathrm{BiOBr}$ structure (JCPDS card no. 09-0393) with a space group of $\mathrm{P} 4 / \mathrm{nmm}(129)$. No characteristic peaks for other impurities can be detected, indicating that phase-pure $\mathrm{BiOBr}$ can be obtained with the three solvent systems. Compared to the HBSMs and BiOBr-IP, the wide diffraction peaks of the BiOBr-GC imply its smaller crystal size and/or poor crystallinity, which should be associated with the high viscosity of the used GC $\left(\sim 934 \mu \mathrm{Pas}\right.$ at $\left.20^{\circ} \mathrm{C}\right)$, much higher than that of IP $(2.37 \mu \mathrm{Pas})$. When the solvent system varies from the GC, GC/IP to IP, the viscosities of the used solvent are reduced in order. As noted, low viscosity is in favor of the higher diffusion rate of ions, generally resulting in higher supersaturation in the solution, which is greatly beneficial for the formation of nuclei [35]. Furthermore, the GC and/or IP probably serve as the soft template to direct the formation of $\mathrm{BiOBr}$ microstructures besides the solvent role. In the single GC system, the number of hydroxyl groups chelated on the surface of $\mathrm{BiOBr}$ nuclei is the largest, inhibiting the growth of the BiOBr crystal, and thus the crystal size of the obtained $\mathrm{BiOBr}-\mathrm{GC}$ would be the smallest. Accordingly, the inhibiting effect of hydroxyl groups upon the growth of $\mathrm{BiOBr}$ crystals is reduced, accompanied by the increased IP content in the mixed solvent, thus speeding up the crystal growth of the BiOBr. In this connection, the samples with even better crystalline is prone to be observed with the solvents ranging from the GC, GC/IP to IP. Simultaneously, the crystal sizes

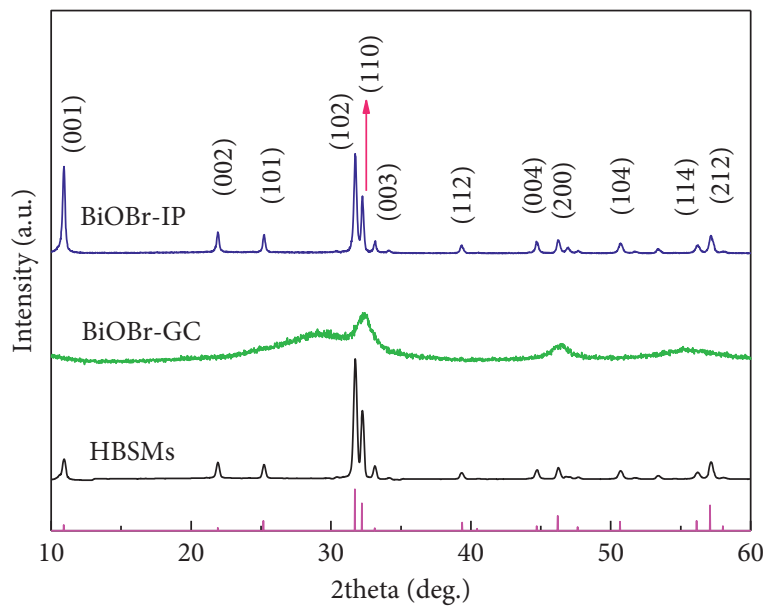

FIGURE 2: XRD patterns of the resultant HBSMs, BiOBr-GC, and BiOBr-IP samples. The magenta vertical lines for the standard spectrum of the BiOBr (JCPDS card no. 09-0393).

of the as-prepared samples were also calculated according to Scherrer formula and collected in Table 1.

The morphology and microstructure of the $\mathrm{BiOBr}$ samples prepared under various conditions are clarified by FESEM analysis, as demonstrated in Figure 3. Figure 3(a) shows the FESEM image of the HBSMs sample. Obviously, the as-prepared HBSMs product consists of a large quantity of well-dispersed submicrospheres with an average diameter of approximately $800 \mathrm{~nm}$. In particular, herein, the asresulted HBSMs with a rough surface are typically hollow, as discerned from some broken microspheres (Figure 3(b)). Inspiringly, the presence of hollow interior guarantees their larger adsorption capacity for contaminants, highlighting its potential applications in waste treatment $[16-19,36]$. In the sole GC medium, the BiOBr-GC mainly displays the spherical structure with the mean diameter of $\sim 500 \mathrm{~nm}$ (Figure 3(c)), which is somewhat smaller than that of HBSMs. Additionally, the BiOBr-IP presents the flake-like structure with a lateral size of $\sim 500 \mathrm{~nm}$ and $\sim 30 \mathrm{~nm}$ in thickness (Figure 3(d)).

The specific microstructures of the HBSMs are further elucidated by the following TEM characterizations. As observed in Figure 4(a), a relatively uniform sphere-like architecture is evident for the resultant HBSMs, which keeps high consistency with the FESEM observations above (Figures 3(a) and 3(b)). The clear contrast between the deeply dark edges and the pale center (Figure 4(a)) visually confirms the hollow feature of the HBSMs [37]. The shell of the HBSMs, which is composed of the nonclose stacking of ultrathin NSs, is estimated as $\sim 100 \mathrm{~nm}$ in thickness (Figure 4(b)). As shown in Figure 4(c), which is taken from 


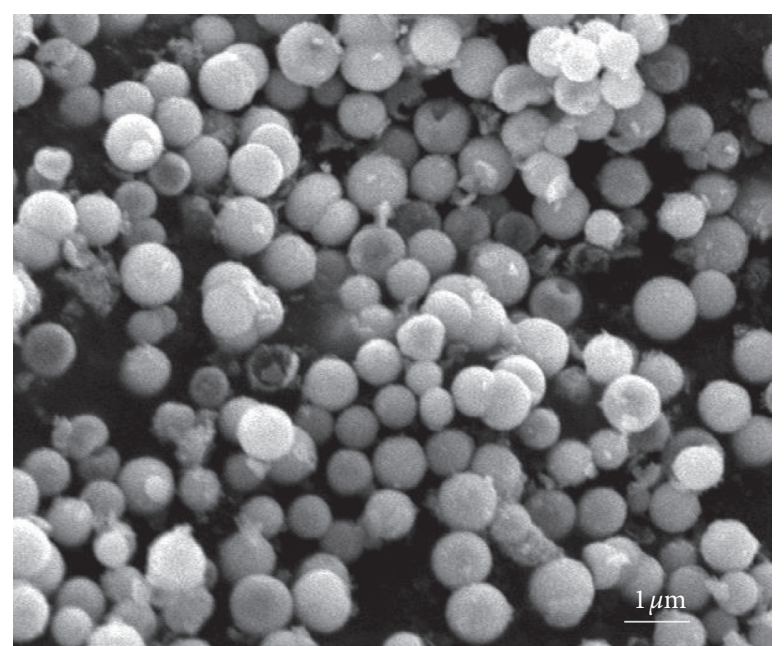

(a)

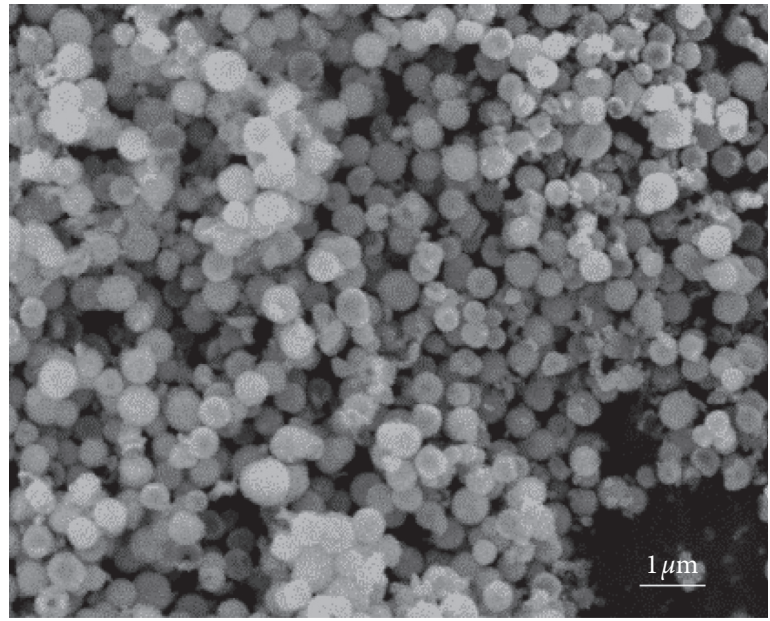

(c)

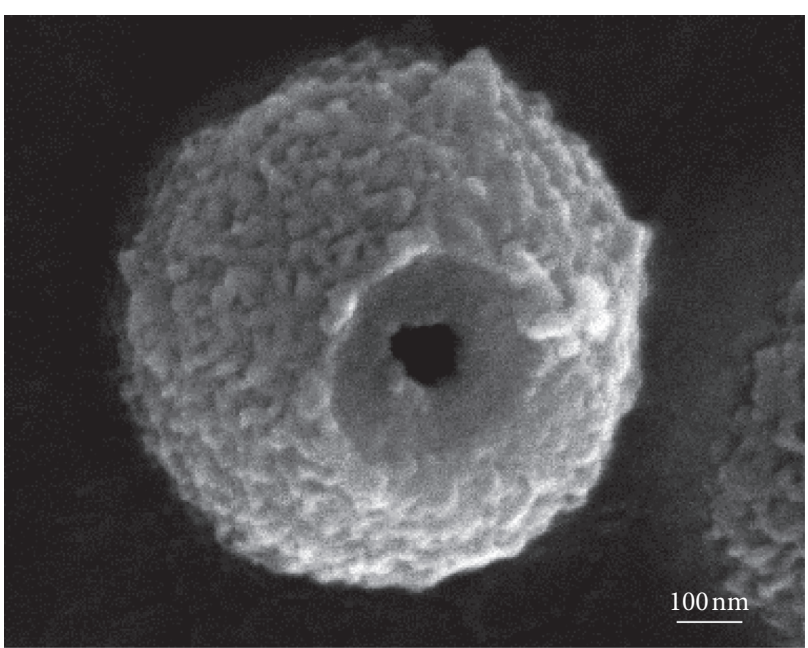

(b)

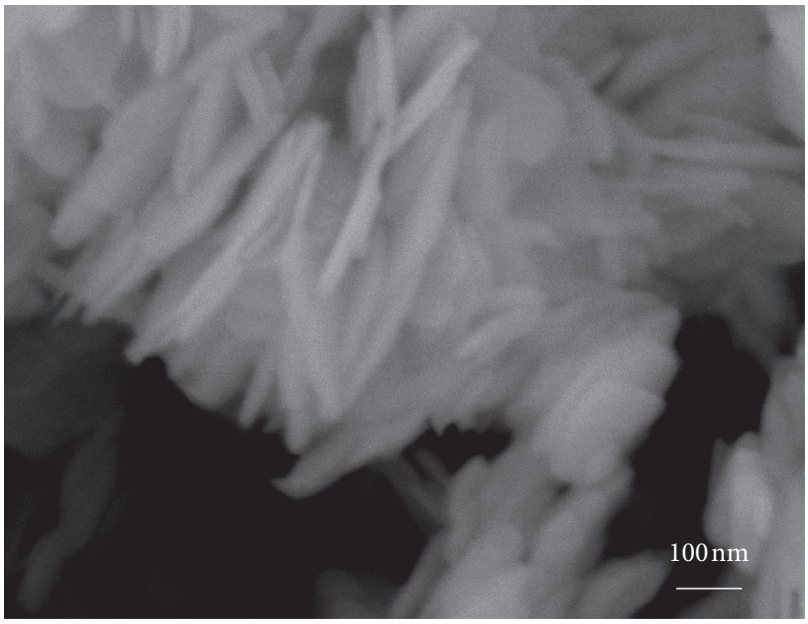

(d)

Figure 3: FESEM images of the (a, b) HBSMs, (c) BiOBr-GC, and (d) BiOBr-IP.

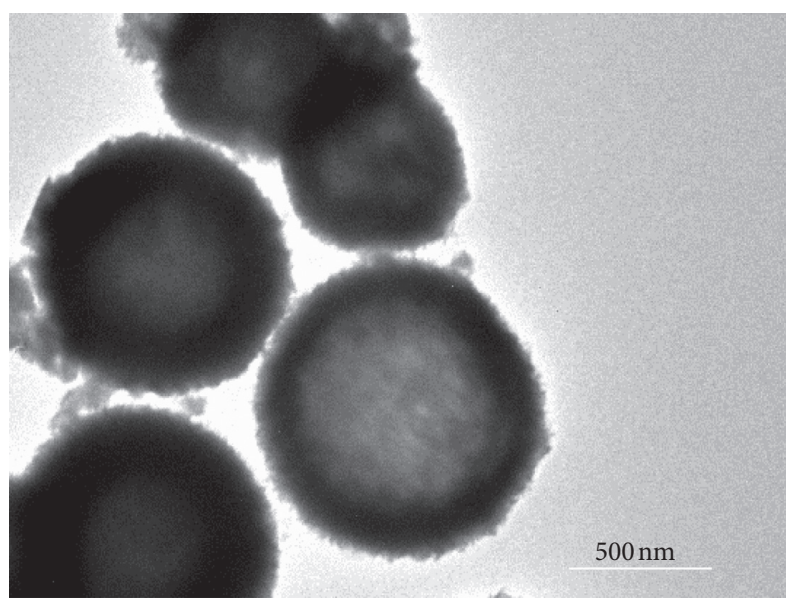

(a)

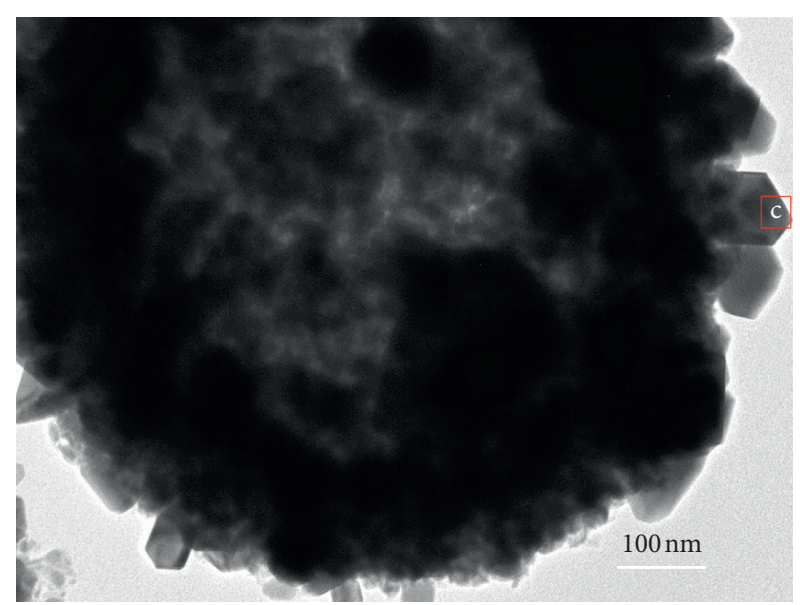

(b)

FIgURE 4: Continued. 


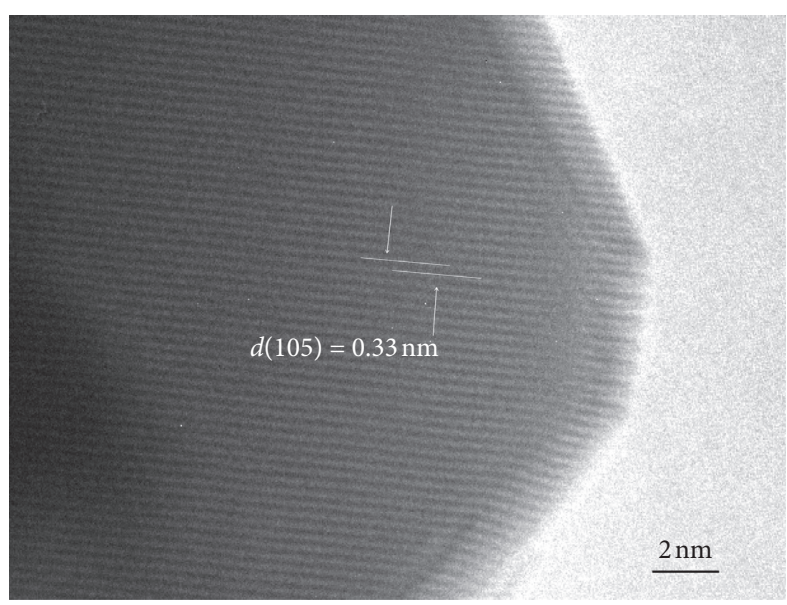

(c)

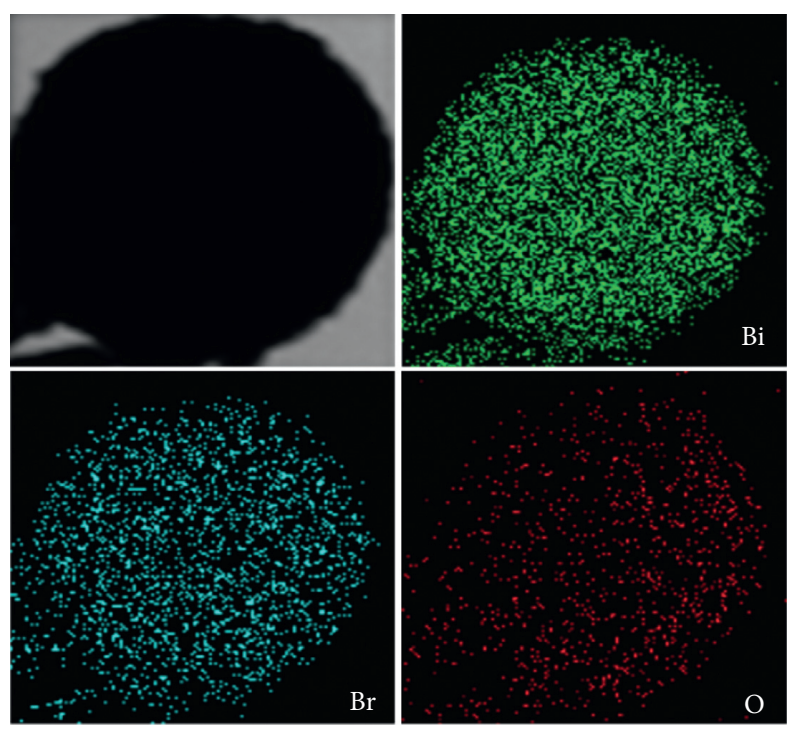

(e)

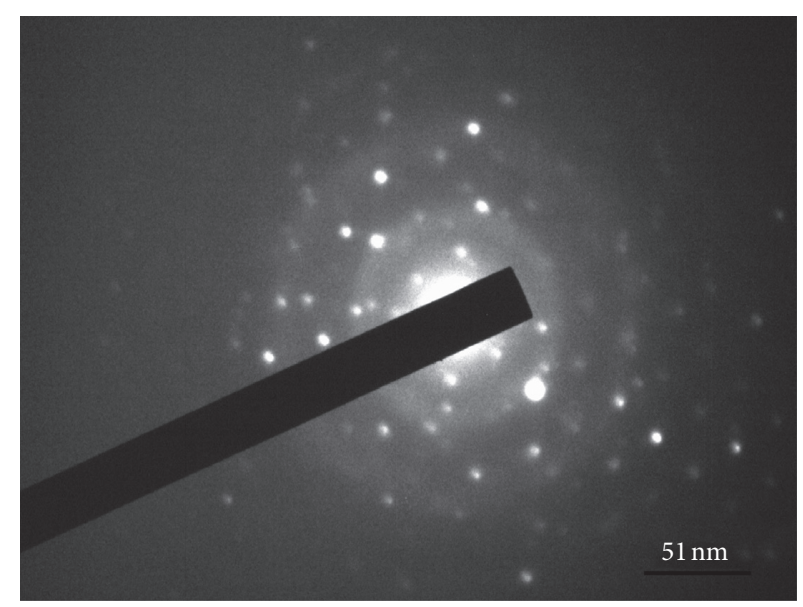

(d)

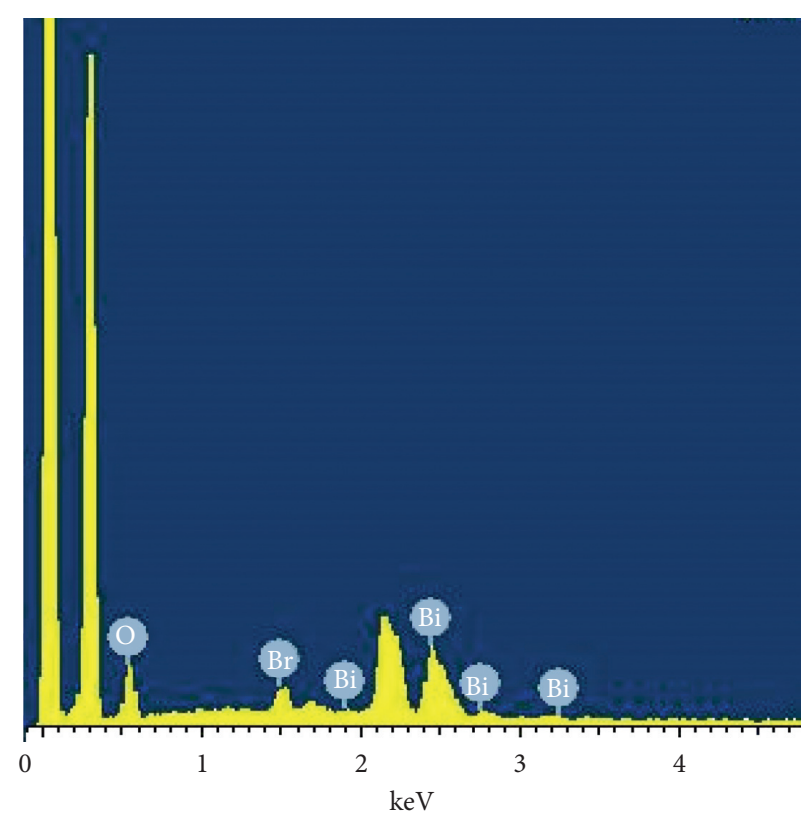

(f)

Figure 4: (a, b) TEM, (c) HRTEM images, (d) SAED pattern, (e) STEM and corresponding elemental (Bi, O, and Br) mapping images, and (f) EDS spectrum of the HBSMs.

the red rectangle region in Figure 4(b), the clear lattice fringes with a spacing of $\sim 0.33 \mathrm{~nm}$ correspond to the interplanar distance of (105) plane of the tetragonal BiOBr. Typical SAED pattern (Figure 4(d)) with a series of concentration rings and some diffraction spots reveals the polycrystalline nature of the HBSMs. Representative STEM and corresponding elemental energy dispersive spectroscopy (EDS) mapping images (Figure 4(e)) prove the uniform distributions of the $\mathrm{Bi}, \mathrm{O}$, and $\mathrm{Br}$ species along the HBSMs architecture. And the elemental ratio of $\mathrm{Bi}$ to $\mathrm{Br}$ is about $1: 1$ for the HBSMs, close to their stoichiometric proportion in the $\mathrm{BiOBr}$ (Figure 4(f)). Thanks to their hollow and nonclose-packed structure, a large BET SSA of $\sim 55.8 \mathrm{~m}^{2} \cdot \mathrm{g}^{-1}$ is obtained for the HBSMs, which is much higher than those of the BiOBr-GC $\left(\sim 17.3 \mathrm{~m}^{2} \cdot \mathrm{g}^{-1}\right)$ and BiOBr-IP $\left(\sim 0.94 \mathrm{~m}^{2} \cdot \mathrm{g}^{-1}\right)$, as summarized in Table 1, further indicating the tremendous influence of solvents upon the BET SSA values. The larger BET surface area and hierarchical hollow properties of the HBSMs would provide more reaction active sites and allow the separating efficiency of the photogenerated $\mathrm{e}^{-} / \mathrm{h}^{+}$pairs, resulting in the enhancement in photocatalytic activities.

Based on the above discussion, a plausible formation mechanism of the HBSMs can be tentatively proposed, as schematically illustrated in Figure 5. Generally, the surfactants are always used to mediate the morphology of materials [37-39]. After being dissolved in the mixed GC/IP solvent, the surfactant DDAB is self-assembled to form the special molecular structure in the mixed solvent through the hydrophobic-hydrophobic interaction. When the $\mathrm{Bi}\left(\mathrm{NO}_{3}\right)_{3} \cdot 5 \mathrm{H}_{2} \mathrm{O}$ is subsequently added to the above mixed solvent, the hydroxyl groups of GC and IP can coordinate with $\mathrm{Bi}^{3+}$ and are further combined with the elemental $\mathrm{Br}$ in 


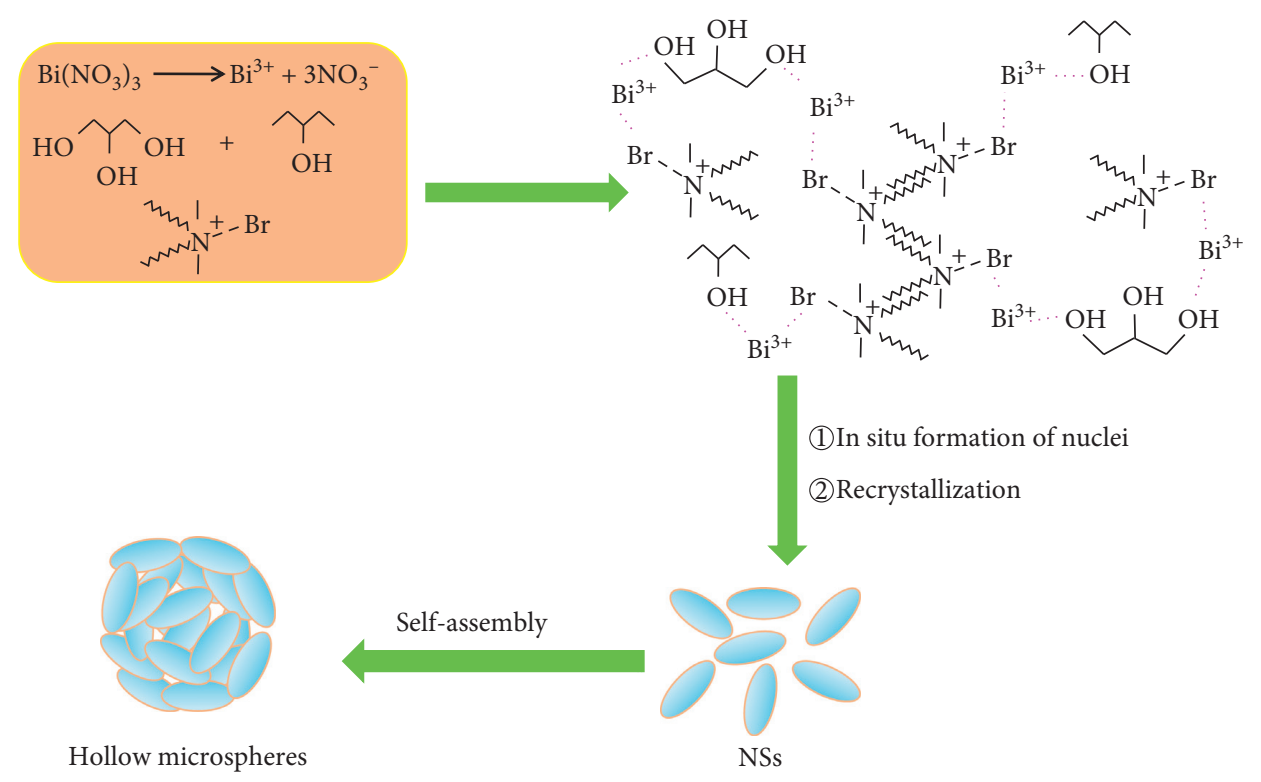

FIgURE 5: Schematic illustration of the formation process of the HBSMs.

the $\mathrm{DDAB}$ molecules through the bridging effect of $-\mathrm{O} \cdots \mathrm{Bi} \cdots \mathrm{Br}$ - to form the complexes. Thus, the concentration of free $\mathrm{Bi}^{3+}$ ions in solution is seriously decreased, which benefits the regulation of the nucleation and growth of crystals during the solvothermal process. The solvothermal reaction environment induces the in situ formation of the $\mathrm{BiOBr}$ nuclei on the DDAB structures, and the newborn $\mathrm{BiOBr}$ nuclei further grow and transform into the NSs through a recrystallization process [29]. Subsequently, the formed NSs are aggregated together and self-assembled into the final HBSMs [40-42]. Further investigations are still on the way to figure out the more exact formation of the HBSMs in our lab.

To further investigate element compositions and chemical states of the HBSMs, XPS analysis was carried out in detail, and the corresponding results are shown in Figure 6. The XPS survey spectrum (Figure 6(a)) demonstrates that the elements of $\mathrm{Bi}, \mathrm{O}, \mathrm{Br}$, and $\mathrm{C}$ coexist in the HBSMs, where the $\mathrm{C}$ signal $(284.6 \mathrm{eV})$ should be attributed to inevitable carbon pollution [36]. In the highresolution spectrum of Bi $4 \mathrm{f}$ (Figure 6(b)), two peaks at binding energies (BEs) of 159.4 and $164.7 \mathrm{eV}$ can be attributed to the $\mathrm{Bi} 4 \mathrm{f}_{7 / 2}$ and $\mathrm{Bi} 4 \mathrm{f}_{5 / 2}$, which indicates the characteristic of $\mathrm{Bi}^{3+}$ in the HBSMs [42]. As seen from the $\mathrm{Br} 3 \mathrm{~d}$ (Figure 6(c)), two peaks at BEs of 68.5 and $69.4 \mathrm{eV}$ are assigned to $\mathrm{Br} 3 \mathrm{~d}_{5 / 2}$ and $\mathrm{Br} 3 \mathrm{~d}_{3 / 2}$, respectively. Meanwhile, as for the $\mathrm{O} 1 \mathrm{~s}$ (Figure 6(d)), the peaks located at 530.4 and $531.7 \mathrm{eV}$ correspond to the crystalline oxygen in the $\mathrm{BiOBr}$ and other excessive oxygen-containing groups (such as, $\cdot \mathrm{O}_{2}^{-}, \mathrm{H}_{2} \mathrm{O}$, etc.) on the surface of the HBSMs sample [43].

In general, the optical absorption property of a semiconductor, together with the migration of electrons, has close relationship with its electronic structure. Thus, it is commonly considered as an important factor to reflect the photocatalytic activities of any semiconductor [44-46]. The $\mathrm{UV}$-vis diffuse-reflectance spectra of the as-prepared $\mathrm{BiOBr}$ samples are displayed in Figure 7(a). Remarkably, the HBSMs specimen illustrates strong photoabsorption properties in the UV light region and even visible light region, indicating its potentially high photocatalytic activities under the visible light irradiation. Compared to that of the HBSMs, the UV-vis absorption spectrum of BiOBr-GC presents a blue shift, which should be related to its relatively smaller crystal size. Besides, a proper red shift in the UV-vis absorption spectrum of the BiOBr-IP may be ascribed to quantum size effect of its nanoscale building blocks [47]. The wavelengths of absorption onset for these $\mathrm{BiOBr}$ samples are all collected in Table 1. According to the absorption spectra (Figure $7(\mathrm{a}))$, the plots of $(\mathrm{Ahv})^{1 / 2}$ versus photon energy $(h v)$ of the BiOBr samples are profiled in Figure $7(\mathrm{~b})$. The $E_{g}$ values can be estimated from the intercepts of the linear region in the plots of $(\mathrm{Ahv})^{1 / 2}$ on the $Y$-axis versus photon energy $(h v)$ on the $X$-axis, as plotted in Figure $7(\mathrm{~b})$. The detailed $E_{g}$ data are exhibited in Table 1. Obviously, the $E_{g}$ decreases from 2.73 to $2.56 \mathrm{eV}$ with the solvent system varying from the GC, GC/IP to IP, which suggests that all these $\mathrm{BiOBr}$ samples have suitable band gaps to be activated by visible light for photocatalytic degradation of organic contaminants.

3.2. Photocatalytic Activities. The photocatalytic activities of the as-prepared $\mathrm{BiOBr}$ samples are purposefully evaluated by the photocatalytic degradation of the MO in water under visible light irradiation and further compared with commercial P25. The degradation efficiencies as a function of reaction time are comparatively illustrated in Figure 8. In the absence of photocatalysts, no obvious degradation of the MO is observed just under visible light irradiation. The adsorption tests were conducted in the dark, and the equilibrium adsorption study reveals that the MO concentration visually underwent slight decrease after the adsorption for $60 \mathrm{~min}$ for all the three photocatalysts. 


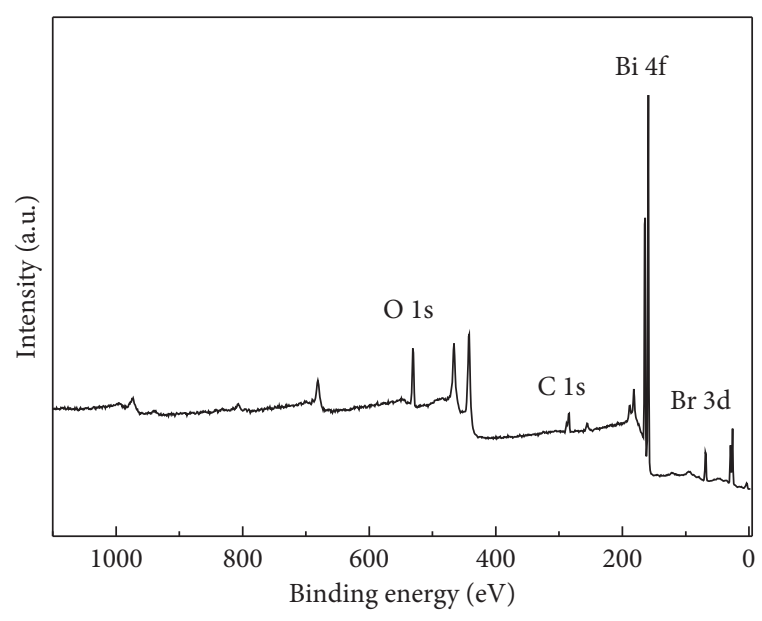

(a)

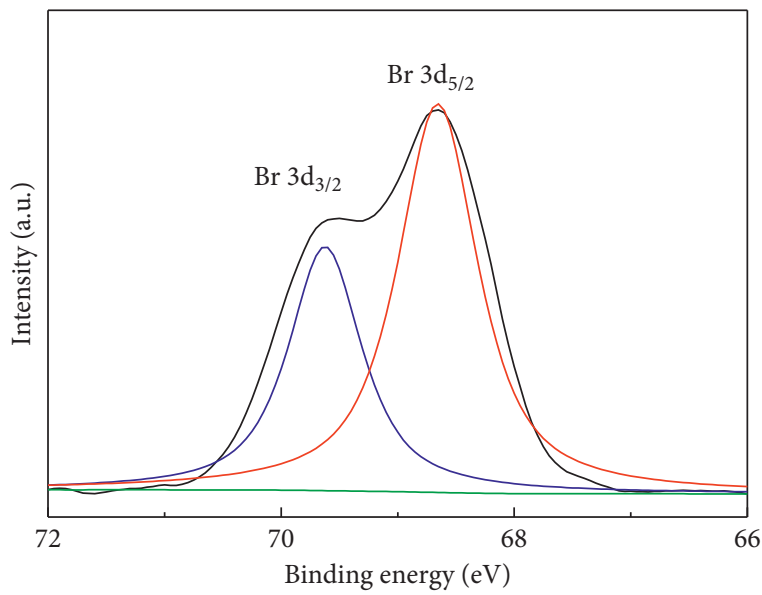

(c)

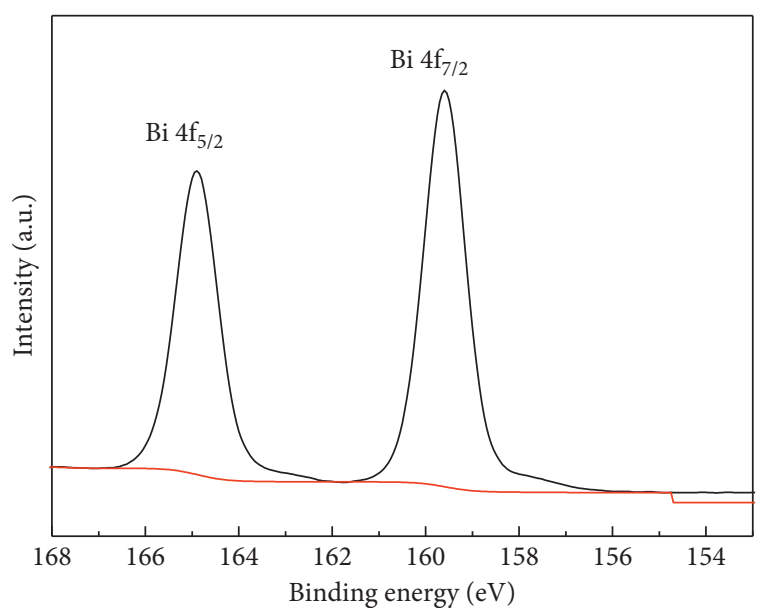

(b)

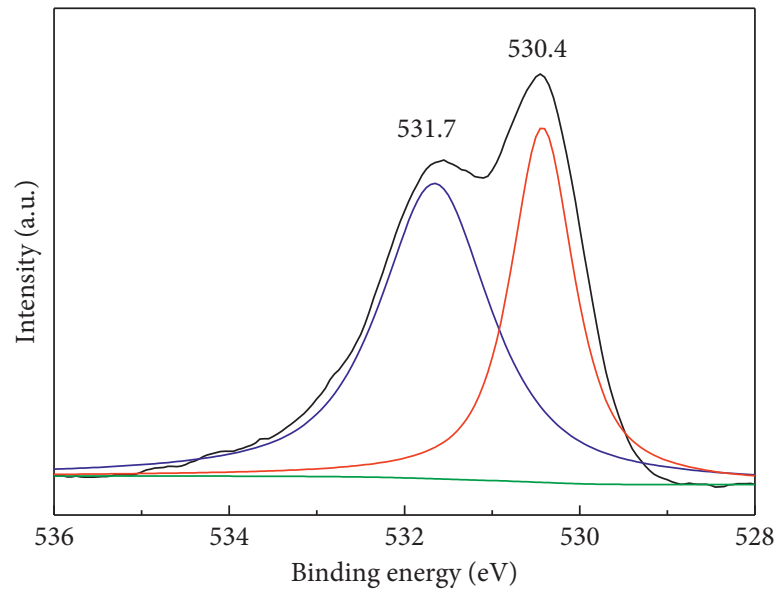

(d)

Figure 6: XPS spectra of the HBSMs: (a) survey, (b) Bi 4f, (c) Br 3d, and (d) O 1s.

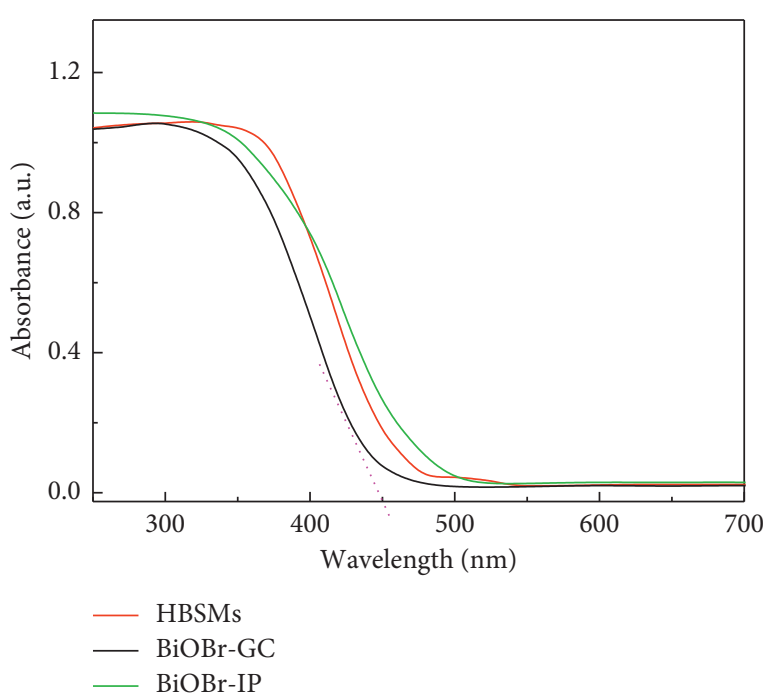

(a)

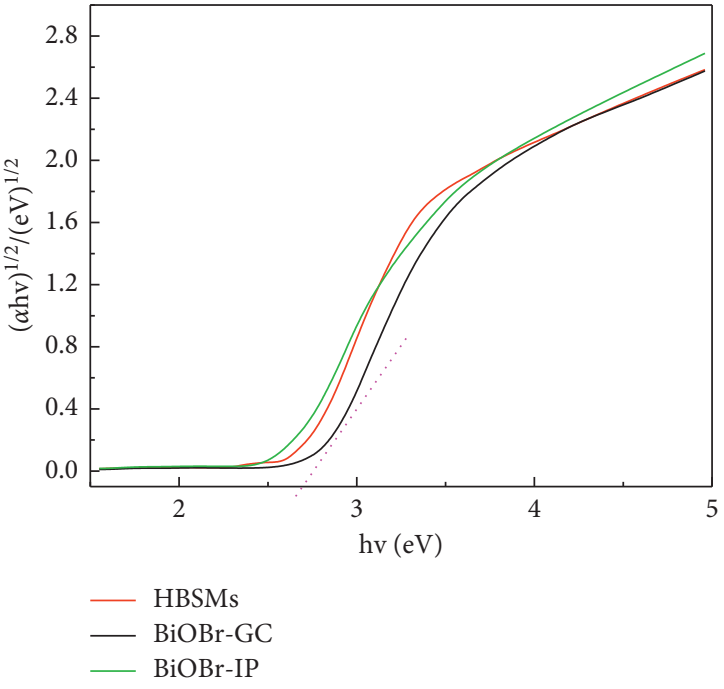

(b)

FIGURE 7: (a) UV-vis absorption spectra and (b) the plot of (Ahv) ${ }^{1 / 2}$ versus photon energy (hv) of the resultant $\mathrm{BiOBr}$ samples as indicated. 


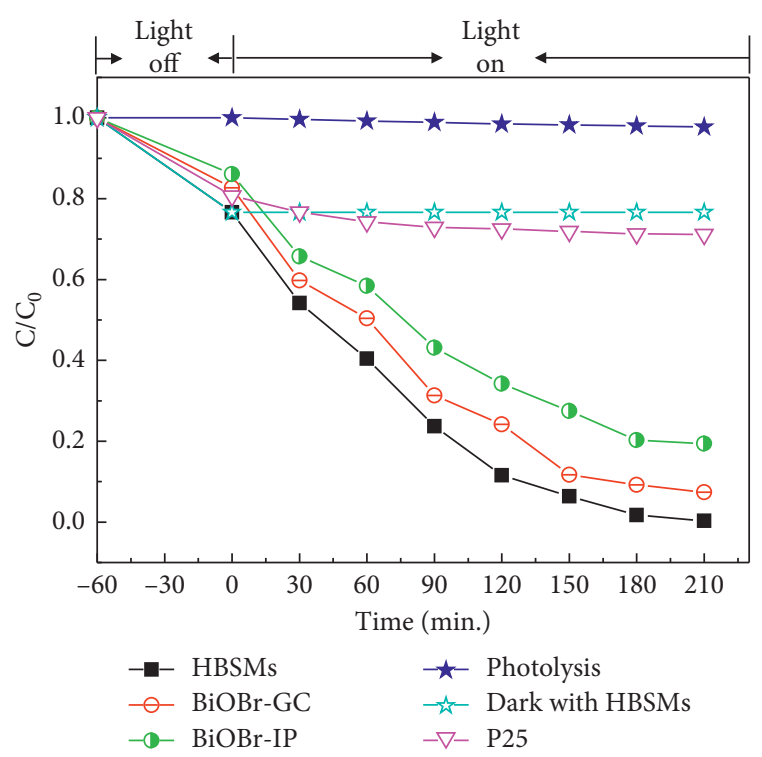

Figure 8: Comparable plots of the MO degradation efficiencies over the as-prepared $\mathrm{BiOBr}$ samples and commercial P25, direct photocatalysis of the MO under visible light irradiation, and the removal of the $\mathrm{MO}$ in dark with the HBSMs. $\mathrm{C}$ and $C_{0}$ indicate the residual and initial concentrations of the $\mathrm{MO}$, respectively.

Corresponding dark adsorption test confirms the strongest adsorption property $(\sim 23.4 \%)$ of the HBSMs over the MO, even stronger than the commercial P25 ( 19.3\%). In spite of relatively weak adsorption capacities of the BiOBr-GC $(\sim 17.3 \%)$ and $\mathrm{BiOBr}-\mathrm{IP}(\sim 13.9 \%)$ for the $\mathrm{MO}$ in comparison to the P25, both of them still demonstrate higher photocatalytic efficiencies than that of the P25 upon visible light irradiation. In particular, the removal rate of the MO by the HBSMs is even close to $\sim 100 \%$ just after visible light irradiation for $210 \mathrm{~min}$, which is much higher than those for the P25 ( 36.1\%), BiOBr-GC ( 92.6\%), and BiOBr-IP ( 80.6\%) under the same conditions. The remarkable photocatalytic activities of the HBSMs can be attributed to the strong adsorption for the pollutant $\mathrm{MO}$ due to its large surface area, the high-efficiency exploitation of visible light owing to its narrower $E_{g}$, and multiple reflections of visible light within the hollow interior, which greatly reduce the recombination of the electron-hole pairs $[48,49]$. Thus, the photoinduced charges are endowed with much longer transport time and more chances to participate in the photocatalytic reactions before their recombination.

To avoid secondary pollution in the practical application, the mineralization ability of HBSMs was also evaluated by monitoring changes in TOC (Figure 9). The results validate its higher mineralisation capacity towards MO. After $210 \mathrm{~min}$ of visible light irradiation, the TOC removal of MO was up to $64.6 \%$, which is slower than the decolorization rate for MO, implying that some MO molecules were actually degraded in spite of the fact that some molecules were directly mineralized to inorganic molecules [50-52].

Generally, some active species, such as hydroxyl radicals $(\cdot \mathrm{OH})$, superoxide radicals $\left(\cdot \mathrm{O}_{2}^{-}\right)$, electrons $\left(\mathrm{e}^{-}\right)$, and holes $\left(\mathrm{h}^{+}\right)$are generated during the photocatalytic degradation of a

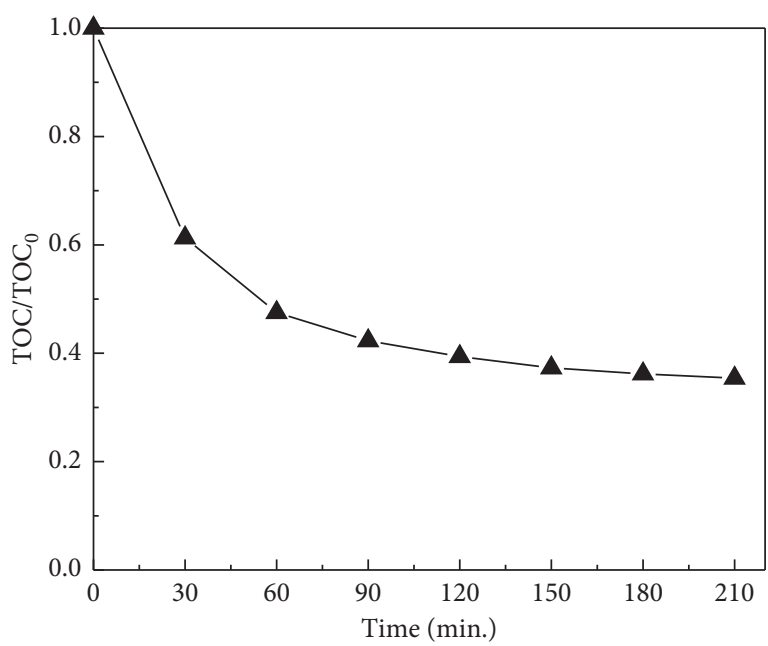

FIgUre 9: Mineralization efficiency of MO by HBSMs.

dye [53-56]. To explore the photocatalytic degradation mechanism of MO over HBSMs, the trapping experiments of main active species were conducted via the addition of scavengers during the photocatalytic reaction. Figure 10 shows the photocatalytic degradation of MO over HBSMs in the absence and presence of scavengers (i.e., IPA for $\cdot \mathrm{OH}$, BQ for $\cdot \mathrm{O}_{2}^{-}$, and EDTA-2Na for $\mathrm{h}^{+}$) under visible light irradiation for $3 \mathrm{~h}$. Obviously, the removal efficiency of $\mathrm{MO}$ decreases dramatically in the systems with the separate addition of BQ or EDTA-2Na, while it is hardly affected by adding IPA into the system, implying that the main active species in the system are $\mathrm{O}_{2}^{-}$and $\mathrm{h}^{+}$.

To determine the flowchart of photogenerated electronhole pairs in HBSMs and further understand the photocatalytic reaction mechanism, the relative band positions of HBSMs were investigated. The positions of conduction band (CB) edge and valence band (VB) edge were calculated via the following empirical equations [57-59]:

$$
\begin{aligned}
& E_{\mathrm{CB}}=x-E_{c}-0.5 E_{g}, \\
& E_{\mathrm{CB}}=E_{\mathrm{VB}}-E_{g},
\end{aligned}
$$

where $x, E_{c}$, and $E_{g}$ are the absolute electronegativity of the photocatalyst $(6.18 \mathrm{eV}$ for $\mathrm{BiOBr}[59,60])$, the free electrons energy $(4.50 \mathrm{eV})$, and the band gap energy, respectively. According to the equation above, the CB and VB of HBSMs can be calculated as 0.35 and $3.01 \mathrm{eV}$, respectively.

Based on the aforementioned analysis, a feasible degradation mechanism of the MO dye by the HBSMs is tentatively proposed, as schematically described in Figure 11. Apparently, owing to the smaller band gap $(2.66 \mathrm{eV})$, the electrons on the VB of HBSMs can be excited to form photoelectrons under the irradiation of visible light and then transferred to the CB of HBSMs, while the generated holes stay in the VB. As noted, the hierarchical hollow feature favors the effective yet efficient separation of the electrons and holes $[16,18]$. Although VB potential of HBSMs $(3.01 \mathrm{eV})$ is more positive than the redox potential of $\cdot \mathrm{OH} /$ $\mathrm{HO}^{-}(1.99 \mathrm{eV})$, the photogenerated $\mathrm{h}^{+}$could hardly oxidize $\mathrm{OH}^{-}$to produce $\cdot \mathrm{OH}$, because the standard redox potential 


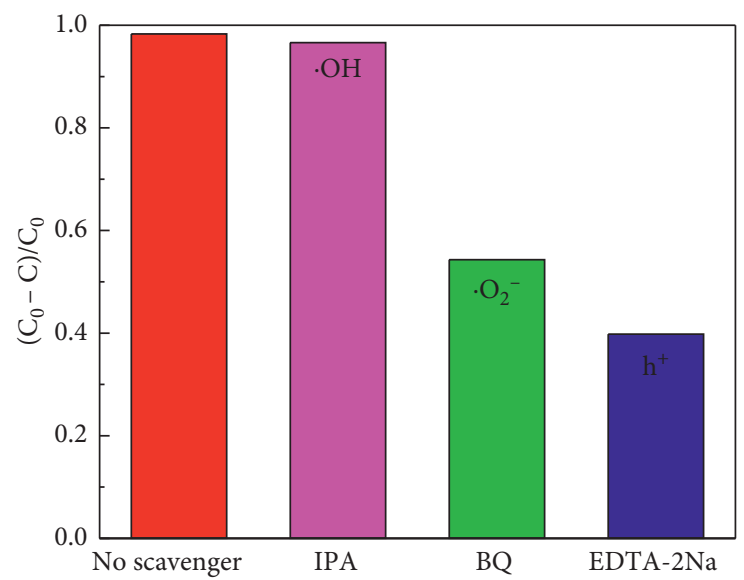

FIgURE 10: Photocatalytic degradation of $\mathrm{MO}$ with $\mathrm{HBSM}$ with the addition of scavengers (IPA for $\mathrm{OH}, \mathrm{BQ}$ for $\cdot \mathrm{O}_{2}^{-}$, and EDTA-2Na for $\mathrm{h}^{+}$) under visible irradiation for $3 \mathrm{~h}$.

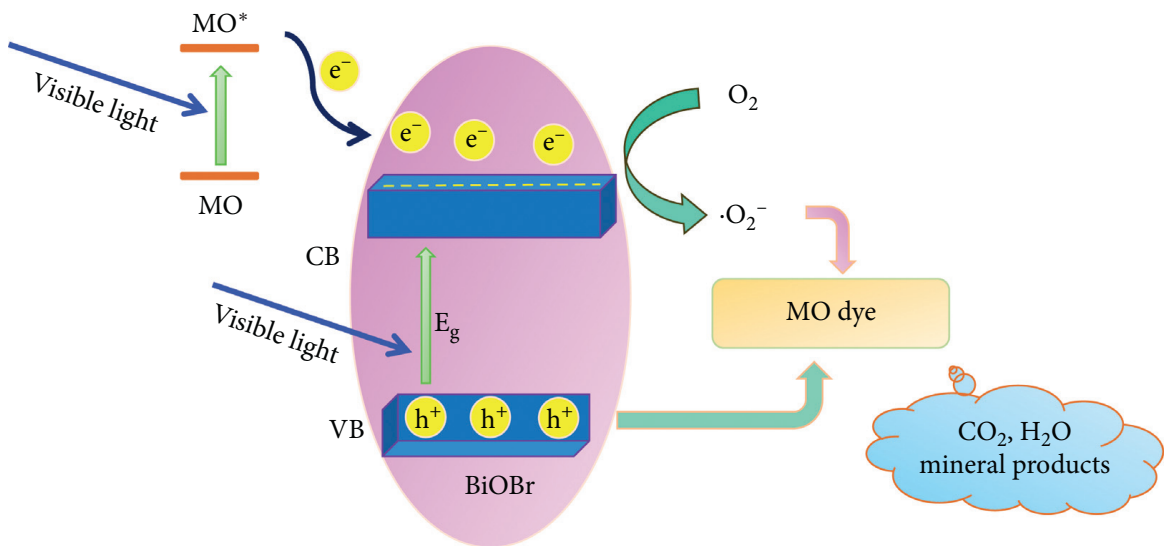

FIGURE 11: Schematic illustration for the photocatalysis mechanism of the MO over the as-obtained HBSMs.

of $\mathrm{Bi}(\mathrm{V}) / \mathrm{Bi}(\mathrm{III})$ is only $1.59 \mathrm{eV}$ [61]. Thus, these holes could not react directly with the $\mathrm{OH}^{-} / \mathrm{H}_{2} \mathrm{O}$ molecules to produce . $\mathrm{OH}$ radicals, while $\mathrm{MO}$ demonstrates much lower redox potential $(1.48 \mathrm{eV})$ [62], suggesting the feasibility of direct oxidation of MO by holes. Simultaneously, the CB edge potential of HBSMs $(0.35 \mathrm{eV})$ was not negative enough to reduce $\mathrm{O}_{2}$ to produce $\cdot \mathrm{O}_{2}^{-}$due to the standard redox potential of $\mathrm{O}_{2} / \cdot \mathrm{O}_{2}^{-}(-0.046 \mathrm{eV})[63,64]$; thus the $\mathrm{e}^{-}$in the $\mathrm{CB}$ of HBSMs could not reduce the adsorpted $\mathrm{O}_{2}$ to generate $\cdot \mathrm{O}_{2}{ }^{-}$. However, the fact that $\cdot \mathrm{O}_{2}{ }^{-}$is the main active substance is not in accordance with the abovementioned trapping experiment. The reason may be related to visible light with more energy $(\lambda>400 \mathrm{~nm}$, energy less than $3.1 \mathrm{eV})$; the photoelectrons may be excited to a higher level of $\mathrm{CB}$ $(-0.09 \mathrm{eV})$. Meanwhile, the MO absorbs the incident photo flux due to the photosensitization phenomenon arising from the transition of MO to MO*. The collected electrons in the higher energy level of CB can be scavenged by the absorbed molecular oxygen on its surface to produce more $\cdot \mathrm{O}_{2}^{-}$owing to the more negative potential $(-0.09 \mathrm{eV})$ than that of $\cdot \mathrm{O}_{2}^{-}$ formation potential $(-0.046 \mathrm{eV})$. Thus, such synergistic effect contributed by electrons in the $\mathrm{CB}$ and holes in the $\mathrm{VB}$ of the HBSMs effectively ensures that the MO is fully oxidized to the $\mathrm{CO}_{2}, \mathrm{H}_{2} \mathrm{O}$, and some mineral products. As a result, the HBSMs demonstrate striking photocatalytic degradation of the dye MO under visible light irradiation.

The excellent reusability of any photocatalyst is also recognized as a key factor for its practical applications. To evaluate the photocatalytic stability of the HBSMs, photocatalytic activities of the HBSMs are investigated by circulating runs in the degradation of the MO under visible light radiation. As can be inferred from Figure 12, the shrinking of degradation rate is still kept within $\sim 4 \%$ even up to six consecutive cycles under the same photocatalytic conditions. In addition, the XRD pattern of HBSMs after six cycles for the photocatalytic degradation of the MO presented no obvious change in comparison with its original pattern (Figure 13), which further confirms the remarkable stability of the resultant HBSMs. The observation here implies that the HBSMs own superior cycling stability, and no obvious photocorrosion takes place during the photocatalytic degradation of the MO molecules, which is of particular significance for its commercial applications. 


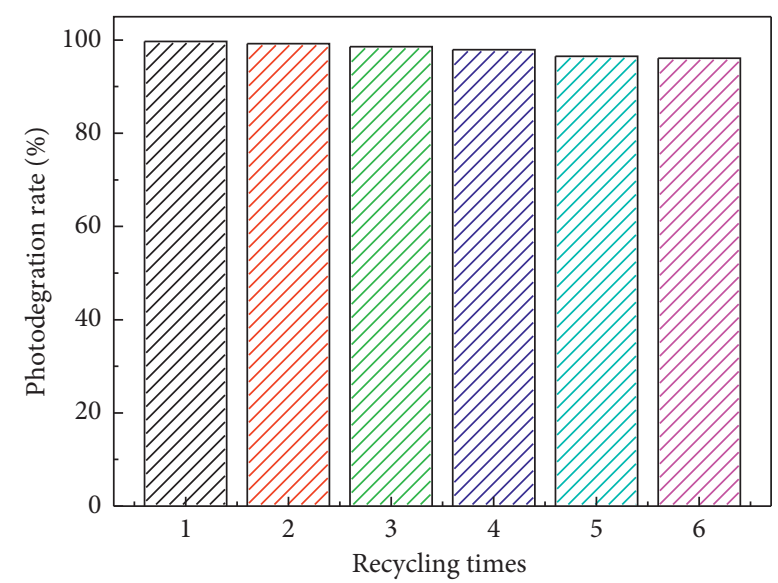

FIGURE 12: Cycling stability of the HBSMs for degradation of the MO.

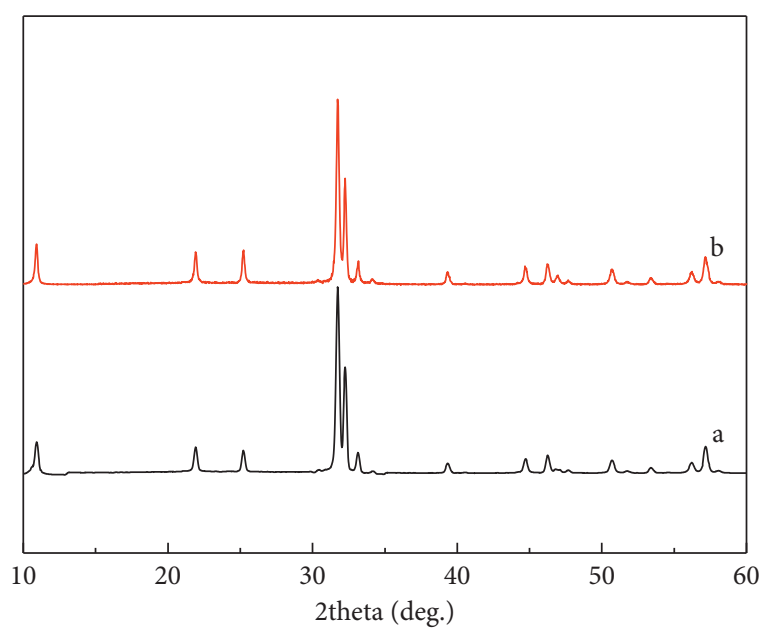

FIgURE 13: XRD spectra of HBSMs (a) and HBSMs after six cycles (b) for the photocatalytic degradation of the MO.

\section{Conclusions}

In summary, the hierarchical hollow $\mathrm{BiOBr}$ submicrospheres were successfully prepared via a facile and efficient solvothermal strategy in a mixed solvent of GC and IP. The key role of the DDAB in the formation of the HBSMs and detailed effects of various solvents on the crystallinity, morphology, and microstructure of the $\mathrm{BiOBr}$ products were investigated. Accordingly, the formation mechanism of the HBSMs was rationally proposed here. More strikingly, the obtained HBSMs demonstrated excellent photocatalytic activity for efficient photodegradation of the organic $\mathrm{MO}$ and possessed good reusability under the visible light radiation, indicating their promising appealing application in waste water treatment. Furthermore, the synthetic methodology presented here can be extended to other hierarchical hollow materials with versatile applications.

\section{Data Availability}

The data used to support the findings of this study are available in Table 1 and Figures 1-13 of this article.

\section{Conflicts of Interest}

The authors declare no conflicts of interest.

\section{Authors' Contributions}

Linrui Hou conceived and designed the experiments; Yawei Niu, Fan Yang, and Fengyue Ge performed the experiments and analyzed the data; Linrui Hou and Changzhou Yuan provided the concept of this research and managed the whole experimental writing process as the corresponding authors; all authors discussed the results and commented on the manuscript.

\section{Acknowledgments}

The authors acknowledge the financial support from National Natural Science Foundation of China (nos. 51772127 and 51772131), Major Program of Shandong Province Natural Science Foundation (no. ZR2018ZB0317), Taishan Scholars (no. ts201712050), and Collaborative Innovation Center of Technology and Equipment for Biological Diagnosis and Therapy in Universities of Shandong.

\section{References}

[1] J. Li, Q. Pei, R. Wang et al., "Enhanced photocatalytic performance through magnetic field boosting carrier transport," ACS Nano, vol. 12, no. 4, pp. 3351-3359, 2018.

[2] C. Mo, J. Jian, J. Li et al., "Boosting water oxidation on metalfree carbon nanotubes via directional interfacial chargetransfer induced by an adsorbed polyelectrolyte," Energy \& Environmental Science, vol. 11, no. 12, pp. 3334-3341, 2018.

[3] Y. Wang, Y. Long, Z. Yang, and D. Zhang, "A Novel ionexchange strategy for the fabrication of high strong BiOI/ $\mathrm{BiOBr}$ heterostructure film coated metal wire mesh with tunable visible-light-driven photocatalytic reactivity," Journal of Hazardous Materials, vol. 351, pp. 11-19, 2018.

[4] H. Liu, Z. Fang, Y. Su et al., "Different atomic terminations affect the photocatalytic nitrogen fixation of bismuth oxybromide: a first principles study," Chemistry-An Asian Journal, vol. 13, no. 7, pp. 799-808, 2018.

[5] R. Li, H. Ren, W. Ma, S. Hong, L. Wu, and Y. Huang, "Synthesis of $\mathrm{BiOBr}$ microspheres with ethanol as self-template and solvent with controllable morphology and photocatalytic activity," Catalysis Communications, vol. 106, pp. 1-5, 2018.

[6] E. Skliri, J. Miao, J. Xie et al., “Assembly and photochemical properties of mesoporous networks of spinel ferrite nanoparticles for environmental photocatalytic remediation," Applied Catalysis B: Environmental, vol. 227, pp. 330-339, 2018.

[7] L. Hou, L. Lian, L. Zhang, T. Wu, and C. Yuan, "Microwaveassisted interfacial hydrothermal fabrication of hydrophobic $\mathrm{CdWO}_{4}$ microspheres as a high-performance photocatalyst," RSC Advances, vol. 4, no. 5, pp. 2374-2381, 2014.

[8] K. Dashtian, M. Ghaedi, H. Shirinzadeh, S. Hajati, and S. Shahbazi, "Achieving enhanced blue-light-driven photocatalysis using nanosword-like $\mathrm{VO}_{2} / \mathrm{CuWO}_{4}$ type II n-n heterojunction," Chemical Engineering Journal, vol. 339, pp. 189-203, 2018.

[9] H. Huang, C. Ma, Z. Zhu et al., "Insights into enhanced visible light photocatalytic activity of $\mathrm{t}$-Se nanorods/ $\mathrm{BiOCl}$ ultrathin 
nanosheets 1D/2D heterojunctions," Chemical Engineering Journal, vol. 338, pp. 218-229, 2018.

[10] L. Hou, H. Hua, S. Liu, G. Pang, and C. Yuan, "Surfactantassisted hydrothermal synthesis of ultrafine $\mathrm{CoMoO}_{4} \cdot 0.9 \mathrm{H}_{2} \mathrm{O}$ nanorods towards high-performance supercapacitors," New Journal of Chemistry, vol. 39, no. 7, pp. 5507-5512, 2015.

[11] $\mathrm{X}$. Su and $\mathrm{D}$. Wu, "Facile construction of the phase junction of $\mathrm{BiOBr}$ and $\mathrm{Bi}_{4} \mathrm{O}_{5} \mathrm{Br}_{2}$ nanoplates for ciprofloxacin photodegradation," Materials Science in Semiconductor Processing, vol. 80, pp. 123-130, 2018.

[12] S. Hong, H. Ren, Y. Fang, Y. Huang, and R. Li, "Template-free solvothermal synthesis of flower-like $\mathrm{BiOBr}$ microspheres in ethanol medium for photocatalytic applications," Russian Journal of Physical Chemistry A, vol. 92, no. 5, pp. 984-991, 2018.

[13] S.-R. Zhu, Q. Qi, Y. Fang, W.-N. Zhao, M.-K. Wu, and L. Han, "Covalent triazine framework modified BiOBr nanoflake with enhanced photocatalytic activity for antibiotic removal," Crystal Growth \& Design, vol. 18, no. 2, pp. 883-891, 2018.

[14] X. A. Dong, W. D. Zhang, Y. J. Sun et al., "Visible-lightinduced charge transfer pathway and photocatalysis mechanism on Bi semimetal@defective BiOBr hierarchical microspheres," Journal of Catalysis, vol. 357, pp. 41-50, 2017.

[15] Y. Wang, J. Sunarso, B. Zhao, C. Ge, and G. Chen, "Onedimensional $\mathrm{BiOBr}$ nanosheets $/ \mathrm{TiO}_{2}$ nanofibers composite: controllable synthesis and enhanced visible photocatalytic activity," Ceramics International, vol. 43, no. 17, pp. 15769-15776, 2017.

[16] S. Demirci, M. Yurddaskal, T. Dikici, and C. Sarığlu, "Fabrication and characterization of novel iodine doped hollow and mesoporous hematite $\left(\mathrm{Fe}_{2} \mathrm{O}_{3}\right)$ particles derived from sol-gel method and their photocatalytic performances," Journal of Hazardous Materials, vol. 345, pp. 27-37, 2018.

[17] Y. Tian, W. Li, C. Zhao, Y. Wang, B. Zhang, and Q. Zhang, "Fabrication of hollow mesoporous $\mathrm{SiO}_{2}$-BiOCl@PANI@Pd photocatalysts to improve the photocatalytic performance under visible light," Applied Catalysis B: Environmental, vol. 213, pp. 136-146, 2017.

[18] Y. J. Zhou, J. W. Shi, D. D. Ma, Z. Y. Fan, C. M. Niu, and L. Z. Wang, "Fabrication of $\mathrm{g}-\mathrm{C}_{3} \mathrm{~N}_{4} / \mathrm{Au} / \mathrm{C}-\mathrm{TiO}_{2}$ hollow structures as visible-light-driven $\mathrm{Z}$-sheme photocatalysts with enhanced photocatalytic $\mathrm{H}_{2}$ evolution," ChemCatChem, vol. 9, no. 19, pp. 3752-3761, 2017.

[19] Y. Peng, J. Xu, T. Liu, and Y. G. Mao, “Controlled synthesis of one-dimensional $\mathrm{BiOBr}$ with exposed (110) facets and enhanced photocatalytic activity," CrystEngComm, vol. 19, no. 43, pp. 6473-6480, 2014.

[20] P. Dumrongrojthanath, T. Thongtem, A. Phuruangrat, and S. Thongtem, "Synthesis and characterization of hierarchical multilayered flower-like assemblies of $\mathrm{Ag}$ doped $\mathrm{Bi}_{2} \mathrm{WO}_{6}$ and their photocatalytic activities," Superlattices and Microstructures, vol. 64, pp. 196-203, 2013.

[21] A. Phuruangrat, A. Maneechote, P. Dumrongrojthanath, N. Ekthammathat, S. Thongtem, and T. Thongtem, "Effect of $\mathrm{pH}$ on visible-light-driven $\mathrm{Bi}_{2} \mathrm{WO}_{6}$ nanostructured catalyst synthesized by hydrothermal method," Superlattices and Microstructures, vol. 78, pp. 106-115, 2015.

[22] X. Han, Q. Chen, H. Zhang, Y. Ni, and L. Zhang, "Template synthesis of $\mathrm{NiCo}_{2} \mathrm{~S}_{4} / \mathrm{Co}_{9} \mathrm{~S}_{8}$ hollow spheres for high-performance asymmetric supercapacitors," Chemical Engineering Journal, vol. 368, pp. 513-524, 2019.

[23] G. Jia, H. You, Y. Song, Y. Huang, M. Yang, and H. Zhang, "Facile synthesis and luminescence of uniform $\mathrm{Y}_{2} \mathrm{O}_{3}$ hollow spheres by a sacrificial template route," Inorganic Chemistry, vol. 49, no. 17, pp. 7721-7725, 2010.

[24] C. Yuan, J. Li, L. Hou et al., "Template-engaged synthesis of uniform mesoporous hollow $\mathrm{NiCo}_{2} \mathrm{O}_{4}$ sub-microspheres towards high-performance electrochemical capacitors," RSC Advances, vol. 3, no. 40, pp. 18573-18578, 2013.

[25] X.-Y. Yu, X.-Z. Yao, T. Luo, Y. Jia, J.-H. Liu, and X.-J. Huang, "Facile synthesis of urchin-like $\mathrm{NiCo}_{2} \mathrm{O}_{4}$ hollow microspheres with enhanced electrochemical properties in energy and environmentally related applications," ACS Applied Materials \& Interfaces, vol. 6, no. 5, pp. 3689-3695, 2014.

[26] L. Liu, Z. Hu, L. Sun, G. Gao, and X. Liu, "Controlled synthesis and enhanced electrochemical performance of Prussian blue analogue-derived hollow $\mathrm{FeCo}_{2} \mathrm{O}_{4}$ nanospheres as lithiumion battery anodes," RSC Advances, vol. 5, no. 46, pp. 36575-36581, 2015.

[27] X. Zhou, X. Li, H. Sun et al., "Nanosheet-assembled $\mathrm{ZnFe}_{2} \mathrm{O}_{4}$ hollow microspheres for high-sensitive acetone sensor," ACS Applied Materials \& Interfaces, vol. 7, no. 28, pp. 15414-15421, 2015.

[28] L. Zhang, X.-F. Cao, X.-T. Chen, and Z.-L. Xue, "BiOBr hierarchical microspheres: microwave-assisted solvothermal synthesis, strong adsorption and excellent photocatalytic properties," Journal of Colloid and Interface Science, vol. 354, no. 2, pp. 630-636, 2011.

[29] J. Xia, S. Yin, H. Li, H. Xu, L. Xu, and Y. Xu, "Improved visible light photocatalytic activity of sphere-like BiOBr hollow and porous structures synthesized via a reactable ionic liquid," Dalton Transactions, vol. 40, no. 19, pp. 5249-5258, 2011.

[30] L. Ye, J. Liu, C. Gong, L. Tian, T. Peng, and L. Zan, "Two different roles of metallic $\mathrm{Ag}$ on $\mathrm{Ag} / \mathrm{AgX} / \mathrm{BiOX}(\mathrm{X}=\mathrm{Cl}, \mathrm{Br})$ visible light photocatalysts: surface plasmon resonance and Z-scheme bridge," ACS Catalysis, vol. 2, no. 8, pp. 1677-1683, 2012.

[31] H. Huang, K. Xiao, Y. He et al., "In situ assembly of BiOI@ $\mathrm{Bi}_{12} \mathrm{O}_{17} \mathrm{Cl}_{2} p$ - $n$ junction: charge induced unique front-lateral surfaces coupling heterostructure with high exposure of BiOI $\{001\}$ active facets for robust and nonselective photocatalysis," Applied Catalysis B: Environmental, vol. 199, pp. 75-86, 2016.

[32] D. He, L. Wang, H. Li, T. Yan, D. Wang, and T. Xie, "Selfassembled $3 \mathrm{D}$ hierarchical clew-like $\mathrm{Bi}_{2} \mathrm{WO}_{6}$ microspheres: synthesis, photo-induced charges transfer properties, and photocatalytic activities," CrystEngComm, vol. 13, no. 12, pp. 4053-4059, 2011.

[33] J. Bi, J. Che, L. Wu, and M. Liu, "Effects of the solvent on the structure, morphology and photocatalytic properties of $\mathrm{Bi}_{2} \mathrm{MoO}_{6}$ in the solvothermal process," Materials Research Bulletin, vol. 48, no. 6, pp. 2071-2075, 2013.

[34] F. Vaquero, R. M. Navarro, and J. L. G. Fierro, "Influence of the solvent on the structure, morphology and performance for $\mathrm{H}_{2}$ evolution of CdS photocatalysts prepared by solvothermal method," Applied Catalysis B: Environmental, vol. 203, pp. 753-767, 2017.

[35] Z. Liu, B. Wu, D. Xiang, and Y. Zhu, "Effect of solvents on morphology and photocatalytic activity of BiOBr synthesized by solvothermal method," Materials Research Bulletin, vol. 47, no. 11, pp. 3753-3757, 2012.

[36] Y. Zhang, Z. Wang, F. U. Zaman et al., "Hollow mesoporous hetero- $\mathrm{ZnO} / \mathrm{ZnMnO}_{3}$ microspheres: template-free formation process and enhanced lithium storage capability towards $\mathrm{Li}$ ion batteries as a competitive anode," Journal of Materials Chemistry A, vol. 7, no. 7, pp. 3264-3277, 2019.

[37] N. G. Macedo, A. F. Gouveia, R. A. Roca et al., "Surfactantmediated morphology and photocatalytic activity of 
$\alpha-\mathrm{Ag}_{2} \mathrm{WO}_{4}$ material," The Journal of Physical Chemistry C, vol. 122, no. 15, pp. 8667-8679, 2018.

[38] Y. Li, Y. Zhao, G. Wu, and J. Zhao, "Facile and efficient synthesis of bismuth nanowires for improved photocatalytic activity," Inorganic Chemistry, vol. 55, no. 10, pp. 4897-4905, 2016.

[39] J. Xia, S. Yin, H. Li, H. Xu, Y. Yan, and Q. Zhang, "Selfassembly and enhanced photocatalytic properties of BiOI hollow microspheres via a reactable ionic liquid," Langmuir, vol. 27, no. 3, pp. 1200-1206, 2011.

[40] Z. Chen, L. Qian, J. Zhu, Y. Yuan, and X. Qian, "Controlled synthesis of hierarchical $\mathrm{Bi}_{2} \mathrm{WO}_{6}$ microspheres with improved visible-light-driven photocatalytic activity," CrystEngComm, vol. 12, no. 7, pp. 2100-2106, 2010.

[41] X.-J. Dai, Y.-S. Luo, W.-D. Zhang, and S.-Y. Fu, "Facile hydrothermal synthesis and photocatalytic activity of bismuth tungstate hierarchical hollow spheres with an ultrahigh surface area," Dalton Transactions, vol. 39, no. 14, pp. 3426-3432, 2010.

[42] Y. Huo, J. Zhang, M. Miao, and Y. Jin, "Solvothermal synthesis of flower-like $\mathrm{BiOBr}$ microspheres with highly visiblelight photocatalytic performances," Applied Catalysis B: Environmental, vol. 111-112, pp. 334-341, 2012.

[43] J. Xu, W. Meng, Y. Zhang, L. Li, and C. Guo, "Photocatalytic degradation of tetrabromobisphenol A by mesoporous $\mathrm{BiOBr}$ : efficacy, products and pathway," Applied Catalysis B: Environmental, vol. 107, no. 3-4, pp. 355-362, 2011.

[44] S.-R. Zhu, M.-K. Wu, W.-N. Zhao et al., "In situ growth of metal-organic framework on $\mathrm{BiOBr} 2 \mathrm{D}$ material with excellent photocatalytic activity for dye degradation," Crystal Growth \& Design, vol. 17, no. 5, pp. 2309-2313, 2017.

[45] B. Wang, J. Durantini, J. Nie, A. E. Lanterna, and J. C. Scaiano, "Heterogeneous photocatalytic click chemistry," Journal of the American Chemical Society, vol. 138, no. 40, pp. 13127-13130, 2016.

[46] S. Pal, A. M. Laera, A. Licciulli, M. Catalano, and A. Taurino, "Biphase $\mathrm{TiO}_{2}$ microspheres with enhanced photocatalytic activity," Industrial \& Engineering Chemistry Research, vol. 53, no. 19, pp. 7931-7938, 2014.

[47] L. Hou, Q. Zhang, L. Ling, C.-X. Li, L. Chen, and S. Chen, "Interfacial fabrication of single-crystalline $\mathrm{ZnTe}$ nanorods with high blue fluorescence," Journal of the American Chemical Society, vol. 135, no. 29, pp. 10618-10621, 2013.

[48] K. Wei, K. Li, L. Yan et al., "One-step fabrication of $\mathrm{g}-\mathrm{C}_{3} \mathrm{~N}_{4}$ nanosheets $/ \mathrm{TiO}_{2}$ hollow microspheres heterojunctions with atomic level hybridization and their application in the multicomponent synergistic photocatalytic systems," Applied $\mathrm{Ca}$ talysis B: Environmental, vol. 222, pp. 88-98, 2018.

[49] M. Waqas, Y. Wei, D. Mao et al., "Multi-shelled $\mathrm{TiO}_{2} / \mathrm{Fe}_{2} \mathrm{TiO}_{5}$ heterostructured hollow microspheres for enhanced solar water oxidation," Nano Research, vol. 10, no. 11, pp. 3920-3928, 2017.

[50] S. S. Imam, R. Adnan, and N. H. Mohd Kaus, "Room-temperature synthesis of flower-like $\mathrm{BiOBr} / \mathrm{Bi}_{2} \mathrm{~S}_{3}$ composites for the catalytic degradation of fluoroquinolones using indoor fluorescent light illumination," Colloids and Surfaces A: Physicochemical and Engineering Aspects, vol. 585, Article ID 124069, 2020.

[51] Z. Liu, G. Wang, and P. Yang, "Selected growth of second phase on $\mathrm{BiOBr}$ facets via spatial charge separation towards enhanced photocatalysis activity," Journal of Industrial and Engineering Chemistry, vol. 66, pp. 262-268, 2018.

[52] D. Majhi, K. Das, A. Mishra, R. Dhiman, and B. G. Mishra, "One pot synthesis of $\mathrm{CdS} / \mathrm{BiOBr} / \mathrm{Bi}_{2} \mathrm{O}_{2} \mathrm{CO}_{3}$ : a novel ternary double Z-scheme heterostructure photocatalyst for efficient degradation of atrazine," Applied Catalysis B: Environmental, vol. 260, Article ID 118222, 2020.

[53] S. Meng, D. Li, M. Sun et al., "Sonochemical synthesis, characterization and photocatalytic properties of a novel cube-shaped $\mathrm{CaSn}(\mathrm{OH})_{6}$," Catalysis Communications, vol. 12, no. 11, pp. 972-975, 2011.

[54] L.-S. Zhang, K.-H. Wong, H.-Y. Yip et al., "Effective photocatalytic disinfection of $E$. coli $\mathrm{K}-12$ using $\mathrm{AgBr}-\mathrm{Ag}-\mathrm{Bi}_{2} \mathrm{WO}_{6}$ nanojunction system irradiated by visible light: the role of diffusing hydroxyl radicals," Environmental Science \& Technology, vol. 44, no. 4, pp. 1392-1398, 2010.

[55] M. Yin, Z. Li, J. Kou, and Z. Zou, "Mechanism investigation of visible light-induced degradation in a heterogeneous $\mathrm{TiO}_{2} /$ eosin Y/rhodamine B system," Environmental Science \& Technology, vol. 43, no. 21, pp. 8361-8366, 2009.

[56] X. Zheng, Q. Yang, S. Huang et al., "Enhanced separation efficiency of photo-induced charge pairs and sunlight-driven photocatalytic performance of $\mathrm{TiO}_{2}$ prepared with the assistance of $\mathrm{NH}_{4} \mathrm{Cl}$," Journal of Sol-Gel Science and Technology, vol. 83, no. 1, pp. 174-180, 2017.

[57] B. Shao, X. Liu, Z. Liu et al., "A novel double Z-scheme photocatalyst $\mathrm{Ag}_{3} \mathrm{PO}_{4} / \mathrm{Bi}_{2} \mathrm{~S}_{3} / \mathrm{Bi}_{2} \mathrm{O}_{3}$ with enhanced visiblelight photocatalytic performance for antibiotic degradation," Chemical Engineering Journal, vol. 368, pp. 730-745, 2019.

[58] H. Yu, B. Huang, H. Wang et al., "Facile construction of novel direct solid-state $\mathrm{Z}$-scheme $\mathrm{AgI} / \mathrm{BiOBr}$ photocatalysts for highly effective removal of ciprofloxacin under visible light exposure: mineralization efficiency and mechanisms," Journal of Colloid and Interface Science, vol. 522, pp. 82-94, 2018.

[59] Z. Ma, L. Deng, G. Fan, and Y. He, "Hydrothermal synthesis of $\mathrm{p}-\mathrm{C}_{3} \mathrm{~N}_{4} / \mathrm{f}-\mathrm{BiOBr}$ composites with highly efficient degradation of methylene blue and tetracycline," Spectrochimica Acta Part A: Molecular and Biomolecular Spectroscopy, vol. 214, pp. 103-110, 2019.

[60] Y. Sun, W. Zhang, T. Xiong et al., "Growth of BiOBr nanosheets on $\mathrm{C}_{3} \mathrm{~N}_{4}$ nanosheets to construct two-dimensional nanojunctions with enhanced photoreactivity for NO removal," Journal of Colloid and Interface Science, vol. 418, pp. 317-323, 2014.

[61] H. Fu, C. Pan, W. Yao, and Y. Zhu, "Visible-light-induced degradation of rhodamine $\mathrm{B}$ by nanosized $\mathrm{Bi}_{2} \mathrm{WO}_{6}$," The Journal of Physical Chemistry B, vol. 109, no. 47, pp. 2243222439, 2005.

[62] Y. Wang, K. Deng, and L. Zhang, "Visible light photocatalysis of BiOI and its Photocatalytic activity enhancement by in situ ionic liquid modification," The Journal of Physical Chemistry C, vol. 115, no. 29, pp. 14300-14308, 2011.

[63] B. Chai, H. Zhou, F. Zhang, X. Liao, and M. Ren, "Visible light photocatalytic performance of hierarchical $\mathrm{BiOBr}$ microspheres synthesized via a reactable ionic liquid," Materials Science in Semiconductor Processing, vol. 23, pp. 151-158, 2014.

[64] D. Zhang, J. Li, Q. Wang, and Q. Wu, "High $\{001\}$ facets dominated $\mathrm{BiOBr}$ lamellas: facile hydrolysis preparation and selective visible-light photocatalytic activity," Journal of Materials Chemistry A, vol. 1, no. 30, pp. 8622-8629, 2013. 